\title{
Aplicabilidade Clínica da Variabilidade da Frequência Cardíaca
}

\author{
Clinical Applications of Heart Rate Variability
}

\section{Polyana Figueiredo Fernandes Lopes ${ }^{1}$, Maria Izabel Bezerra de Oliveira ${ }^{2}$, Samanta Max de Sousa André ${ }^{2}$, Daiany Laise Araújo do Nascimento ${ }^{2}$, Cynthia Sara de Souza Silva Gleidson Mendes Rebouças ${ }^{4}$, Thiago Renee Felipe ${ }^{5}$, Nailton José Brandão de Albuquerque Filho ${ }^{6}$, Humberto Jefferson de Medeiros ${ }^{7}$}

\section{RESUMO}

Objetivo. O objetivo deste trabalho foi revisar aspectos conceituais da Variabilidade da Frequência Cardíaca (VFC), seus dispositivos de mensuração, índices utilizados para sua análise e utilização clínica. Método. Uma revisão de literatura não sistemática realizada através de busca eletrônica conduzidas nas seguintes bases de dados: Medline/ PubMed, Lilacs, Ovid, Science Direct e Biomed Central acerca da utilização clínica da VFC. Resultados. A VFC compreende as oscilaçóes entre os intervalos RR que refletem as modificaçóes na frequência cardíaca em função da atuação conjunta das divisóes simpática e parassimpática do sistema nervoso autônomo. Sua análise pode ser executada em função de dois parâmetros: domínio de tempo e domínio de frequência. O eletrocardiograma, conversores analógicos e cardiofrequencímetros são os principais instrumentos utilizados para obtenção de seus índices, onde a alta variabilidade indica boa adaptação fisiológica do organismo, enquanto que sua redução tem sido apontada como importante indicador para o surgimento de patologias ou de complicaçóes em pacientes com doenças de base conhecidas. Conclusâo. O estudo da VFCvem se apresentando como importante ferramenta de avaliação do funcionamento do organismo em condiçôes normais e patológicas proporcionando assim o desenvolvimento de açôes pelos profissionais da saúde visando prevenção e/ou detecção de várias fisiopatologias.

Unitermos. Frequência Cardíaca, Adaptação Fisiológica, Sistema Nervoso Autônomo.

Citaçáo. Lopes PFF, Oliveira MIB, Sousa AndréSM, Nascimento DLA, Silva CSS, Rebouças GM, Renee Felipe T, Albuquerque Filho NJB, Medeiros HJ. Aplicabilidade Clínica da Variabilidade da Frequência Cardíaca.

1.Bacharel em Enfermagem e Aluna do Curso de Bacharelado em Nutrição do Centro Universitário do Rio Grande do Norte - UNI-RN, Natal-RN, Brasil; 2.Aluna do Curso de Bacharelado em Nutrição do Centro Universitário do Rio Grande do Norte - UNI-RN, Natal-RN, Brasil; 3.Aluna do Curso de Licenciatura em Educaçáo Física do Centro Universitário do Rio Grande do Norte - UNI-RN, Natal-RN, Brasil; 4.Licenciado Pleno em Educaçâo Física pela Universidade do Estado do Rio Grande do Norte -UERN,Especialista em Fisiologia do Exercício. Professor do Centro Universitário do Rio Grande do Norte - UNI-RN, Natal-RN, Brasil; 5.Bacharel em Educaçăo Física pela Universidade Potiguar - UNP. Programa de Pós-graduação Stricto Sensu em Saúde e Sociedadeda Universidade do Estado do Rio Grande do Norte - UERN, Mossoró-RN, Brasil; 6.Bacharel em Educaçáo física pela Universidade Potiguar - UNP. Programa de Pós-graduação Stricto Sensu em Saúde e Sociedadeda Universidade do Estado do Rio Grande do Norte - UERN, Mossoró-RN, Brasil; 7.Licenciado Pleno em Educação Física pela Universidade Federal do Rio Grande do Norte - UFRN, Doutor em Ciências da Saúde pela Universidade Federal do Rio Grande do Norte - UFRN. Afiliação profissional: Professor do Programa de Pós Graduaçáo em Saúde e Sociedade da Universidade do Estado do Rio Grande do Norte - UERN, Mossoró-RN, Brasil.

\section{ABSTRACT}

Objective. The purpose of this study was to review the conceptual aspects of Heart Rate Variability (HRV), its measurement devices, indexes used for analysis and clinical use. Method. A non-systematic literature review conducted through electronic search conducted in the following databases: Medline/PubMed, Lilacs, Ovid, Science Direct and BioMed Central about the clinical use of HRV. Results. HRV comprises oscillations between RR intervals that reflect the changes in heart rate due to the joint action of the sympathetic and parasympathetic divisions of the autonomic nervous system. The HRV's analysis can be performed by the basis of two parameters: the time domain and frequency domain. The electrocardiogram, heart monitors and analog converters are the main instruments used to obtain indices that allow the assessment of HRV, whose high variability indicates good physiological adaptation of the organism while its reduction has been identified as an important indicator for the emergence of pathologies or complications in patients with underlying diseases known. Conclusion. The study of HRV has been presented as an important tool for assessing the body functioning in normal and pathological conditions thus providing the development of actions by health professionals aiming prevention and/or detecting several physiopathologies.

Keywords. Heart Rate, Physiological Adaptation, Autonomic Nervous System.

Citation. Lopes PFF, Oliveira MIB, Sousa AndréSM, Nascimento DLA, Silva CSS, Rebouças GM, Renee Felipe T, Albuquerque Filho NJB, Medeiros HJ. Clinical Applications of Heart Rate Variability.

Trabalho realizado pelo Grupo de Estudos em Modulaçáo Autonômica do Coraçáo (GEMAC) do Centro Universitário do Rio Grande do Norte, Natal-RN, Brasil.

Endereço para correspondência: Gleidson M Rebouças

Rua Teofilândia, 265, Bloco C, apto 202, Bairro Neópolis CEP 59088380, Natal-RN, Brasil. Tel.: (84) 8801-4772 E-mail: gleidsonmr@yahoo.com.br 


\section{INTRODUÇÃO}

O coração é um órgão que apresenta células com ritmicidade própria, capazes de gerar potenciais de ação, responsáveis pelo estabelecimento da frequência cardíaca cujo controle é feito, em parte pelo Sistema Nervoso Autônomo (SNA). Este por sua vez está dividido em uma área denominada simpática que atua sobre o miocárdio e uma parassimpática cuja atuação se dá sobre o nó sinoatrial, miocárdio atrial e o nó atrioventricular. A atuação dessas vias configura-se de forma antagônica, onde a ação simpática promove o aumento da frequência cardíaca enquanto a parassimpática promove a sua diminuição. Por essa razão, modificaçôes na frequência cardíaca são esperadas como respostas normais do organismo a estímulos fisiológicos e ambientais tais como: respiração, exercício físico, estresse, alterações hemodinâmicas, metabólicas, sono e desordens induzidas por doenças ${ }^{1}$. A descoberta da relação entre o sistema nervoso autônomo e mortalidade por doenças cardiovasculares tornaram necessária à realização de estudos acerca do aumento da atividade simpática e redução da atividade parassimpática, condição essa encontrada em diversas patologias do sistema cardiovascular, e o desenvolvimento de marcadores quantitativos da atividade autonômica cardíaca, sendo aVariabilidade da Frequência Cardíaca (VFC) o marcador mais promissor. A VFC compreende as oscilaçóes entre os intervalos $\mathrm{RR}$ dos batimentos cardíacos que refletem as modificaçôes resultantes da atuação do SNA sobre o comportamento da frequência cardíaca ${ }^{2,3}$. Por ser uma ferramenta não invasiva e de fácil utilização teve seu uso largamente difundido, porém por apresentar diversos índices a sua compreensão mostrou-se muito mais complexa, apesar da aparente facilidade na sua utilização. A grande quantidade de trabalhos realizados e a complexidade apresentada na análise dos seus índices incentivou a Sociedade Europeia de Cardiologia e Sociedade Americana de Estimulação Cardíaca e Eletrofisiologia formarem uma força tarefa com o objetivo de padronizar a nomenclatura utilizada, os seus índices e seus métodos de mensuração ${ }^{4}$. Após sua padronização tornou-se uma ferramenta que tem semostrado eficiente na análise da função autonômica cardíaca em indivíduos saudáveis, atletas além de portadores de diversas doenças ${ }^{1,5}$. O objetivo do presente trabalhofoi realizar uma revisãosobre aspectos conceituais da VFC, identificar seus dispositivos de mensuração, índices utilizados para sua análise e mostrar a sua utilização clínica.

\section{Dispositivos de Mensuraçáo}

O eletrocardiograma (ECG), conversores analógicos e cardiofrequencímetros são os principais instrumentos utilizados para obtenção de índices que possibilitam a análise da VFC. Dentre esses dispositivos os cardiofrequencímetros vem se destacando devido a sua fácil aplicação, natureza não invasiva e a possibilidade do seu uso em estudos de campo e durante a prática desportiva permitindo assim registros da VFC com segurança e praticidade fora do ambiente ambulatorial. O ECG, apesar de ainda ser bastante utilizado, trás algumas limitações no seu uso devido à necessidade da sua utilizaçáo dentro do ambiente ambulatorial, seu alto custo e uma alta complexidade em seu uso tais como os numerosos eletrodos que precisam ser posicionados em pontos específicos no indivíduo para que os registros ocorram adequadamentealém da necessidade do paciente permanecer em repouso ${ }^{6}$. Porém, é válido salientar que apesar da facilidade oferecida pelo cardiofrequencímetro, ele não substitui o uso do ECG em exames cardiológicos?.

\section{Métodos Lineares de Avaliação da Variabilidade da Frequência Cardíaca}

As análises da VFC, a partir de métodos lineares, podem ser executadas em função de dois parâmetros; domínio de tempo e domínio de frequência ${ }^{8}$. O domínio do tempo realiza análises com registros que derivaram de tempos superiores a1 0 minutos, expressos em milissegundos, através do qual se determina a variação de duração dos intervalos entre os complexos QRS normais resultantes da despolarização sinusale seus índices matemáticossão ${ }^{5,8,9}$ : SDNN (Standard Deviation of all normal NN interval), desvio padrão de todos os intervalos RR normais gravados em um intervalo de tempo; SDANN (Standart Deviation of the Average NN Interval), representa o desvio padrão das médias dos intervalos RR normais, a cada 5 minutos, em um intervalo de tempo; SDNNi (The Mean of the 5 minutes Standard Deviation of NN Intervals) é a média do desvio padrão dos intervalos $\mathrm{RR}$ normais a cada 5 minutos; rMSSD (Root-Mean of square sucessive $N N$ interval difference), é a raiz quadrada da média do 
quadrado das diferenças entre intervalos $\mathrm{R} R$ normais adjacentes, em um intervalo de tempo e pNN50 (Percent of normal-normal NN intervals whose difference exceeds 50 $m s)$, representa a porcentagem dos intervalos RR adjacentes com diferenças de duração maior que $50 \mathrm{~ms}$. SDNN, SDANN, SDNNi são índices obtidos através de intervalos RR individuais e representam as atividades simpática e parassimpática, enquanto que, rMSSD e pNN50 são obtidos através de intervalos $R R$ adjacentes e refletem a atividade parassimpática.

O domínio da frequência utiliza registros da intensidade das ondas verificadas em intervalos de tempo menores tendo sido verificado na literatura intervalos de tempo de até 4 segundos, sua unidade de medida é o Hertz e seus componentes são1,10: HF (High Frequency): variação de 0,15 a $0,4 \mathrm{~Hz}$ que corresponde à modulação respiratória e é um indicador da atuação do nervo vago sobre o coração; LF (Low Frequency): variação entre 0,04 e $0,15 \mathrm{~Hz}$, decorrente da ação conjunta dos componentes parassimpático e simpático sobre o coração, com predominância do simpático; VLF (Very Low Frequency) e ULF (Ultra Low frequency): são índices menos utilizados por não ter explicação fisiológica bem estabelecida.

\section{Aplicabilidade Clínica da VFC}

A obtenção dos índices da VFC é de extrema importância para a compreensão clínica acerca de determinadas variáveis fisiológicas, pois aumentos na variabilidade indicam boa adaptação fisiológica do organismo e de sua manutenção pressupondo uma condição de estabilidade do sistema biológico ao passo que reduçóes têm sido apontadas como preditores de doenças ou do aparecimento de eventos adversos em pacientes com doençasjá conhecidas ${ }^{1,11}$. São utilizados tambémpara melhor compreensão darelação de certas doenças e a modulação autonômica.

Em estudo com mulheres diagnosticadas com Anorexia Nervosa, os autores encontraram uma redução da frequência cardíaca, uma baixa VFC, redução no componente $\mathbf{L F}$ e elevação no $\mathbf{H F}$ sugerindo uma disfunção cardíaca autonômica na forma de anormalidades no controle vagal cardíaco ${ }^{12}$; $\mathrm{Na}$ epilepsia a redução da VFC, durante o período interictal, é caracterizado por diminuição de HF, rMSSD, pNN50 e aumento da relação LF/
HF, refletindo a redução do tônus vagal cardíaco ${ }^{13}$; Em outro estudo com sujeitos asmáticos,foram encontrados aumento do $\mathbf{H F}$ em relação ao $\mathbf{L F}$ e uma diminuição da relação LF/HF ${ }^{14}$; Sujeitos com Distúrbios de Ansiedade, apresentam os índices SDNN, rMSSD, pNN50, LF e HF reduzidos quando comparados aos indivíduos que não apresentam esses distúrbios ${ }^{15}$; Em amostra de Obesos os autores apontaram para um aumento de $10 \%$ na gordura corporal e associaram o ganho de peso com a diminuição da atividade parassimpática, enquanto que a perda de peso tem influência direta sobre a atividade autonômica do coração refletidos no aumento da HF, VLF, SDNN e SDANN após a perda de peso ${ }^{10}$.

Estudos com amostras que apresentam fisiopatologias cardíacas diagnosticadas têm reportado as alteraçóes na modulação autonômica. Amostras com cardiopatia congênita os pacientes apresentam uma redução nos índices SDNN e rMSSD, no que diz respeito ao domínio do tempo, e redução nos índices do domínio da frequência HF e LF representando assim uma redução da VFC e maior probabilidade da ocorrência de eventos adversos ${ }^{16}$. Estudo com sujeitos pós Infarto Agudo do Miocárdio (IAM) e Dislipidemias apresentou redução dos índices SDANN e SDNN, nos casos de IAM e apenas do SDANN nas dislipidemias ${ }^{8}$; Em amostras com Diabetes a degeneração neurológica periférica, comum na referida patologia, afeta as divisôes simpática e parassimpática do sistema nervoso, assim como o inadequado controle metabólico são fatores importantes causadores da redução da VFC, que nesses pacientes tem sua redução refletida nos índices $\mathbf{H F}$ e $\mathbf{L F}^{17,18}$; Em indivíduos com Hipertensão Arterial os índices SDNN, rMSSD, pNN50, HF, LF, LF/ HF encontram-se reduzidos quando comparados a pacientes normotensos ${ }^{1}$; por fim, estudos recentes têm mostrado que na Esquizofrenia a VFC encontra-se reduzida, com redução de HF, rMSSD, SDANN e aumento da relação $\mathbf{L F} / \mathbf{H F}$, tanto em pacientes em tratamento medicamentoso quanto nos que não estão sob tratamento ${ }^{19,20}$.

\section{CONCLUSÃO}

Apesar da necessidade de mais estudos acerca da aplicabilidade da VFC, no que diz respeito à uma melhor compreensão e padronização das alterações autonômicas em desordens crônicas como as cardiovasculares, respira- 
tórias, metabólicas e neurológicas, por exemplo. A VFC vem se apresentando como um instrumento náo invasivo, de avaliação da modulação autonômica em condiçóes normais e patológicas, com índices e dispositivos próprios de mensuração. De fácil aplicação e obtenção dos dados configura-se em um preditor de doenças e agravos em condiçóes patológicas já existentes. Sendo assim, seu estudo contribuirá para a compreensão da ligação entre as funçóes cardíacas e neurais, apresentando-se como uma nova ferramenta de auxílio no diagnóstico clínico de patologias e de extrema utilidade para o desenvolvimento de açôes dos profissionais da saúde, visando prevenção el ou detecção dos males que acometem o sujeito. Devido a sua natureza não invasiva configura-se em uma técnica atraente não só em relação à avaliação de populações adultas em situaçóes estáticas como também em crianças e sujeitos de um modo geral em situaçôes dinâmicas.

\section{REFERÊNCIAS}

1.Vanderlei LCM, Pastre CM, Hoshi RA, Carvalho TD, Godoy MF. Noçôes básicas de variabilidade da frequência cardíaca e sua aplicabilidade clínica. Rev Bras Cir Cardiovasc 2009;24(2):205-17.

http://dx.doi.org/10.1590/S0102-76382009000200018

2.Task Force of the European Society of Cardiology and the North American Society of Pacing and Electrophysiology. Heart rate variability: standar$\mathrm{d}$ s of measurement, physiological interpretation and clinical use. Circulation 1996;93(5):1043-65.

http://dx.doi.org/10.1161/01.CIR.93.5.1043

3.Rajendra AU, Paul JK, Kannathal N, Lim CM, Suri JS. Heart rate variability: a review. Med Bio Eng Comput 2006;44(12):1031-51.

http://dx.doi.org/10.1007/s11517-006-0119-0

4.Portella RB. Disfunção na regulação autonômica vagal cardíaca em pacientes com hipertireoidismo subclínico sem cardiopatia aparente [dissertação].Rio de Janeiro: Universidade Federal do Rio de Janeiro; 2006, 63p.

5.Mark L R, Chad M T, Christian A O, Thai V, Kenneth GP. Clinical Applications of Heart Rate Variability in the Triage and Assessment of Traumatically Injured Patients. Anesthesiol Res Pract 2011;2011:1-8.

6.Porto LGG, Junqueira JLF. Comparison of Time-Domain Short-Term Heart Interval Variability Analysis Using a Wrist-Worn Heart Rate Monitor and the Conventional Electrocardiogram. Pacing Clin Electrophysiol 2009;32:43-51. http://dx.doi.org/10.1111/j.1540-8159.2009.02175.x
7.Pimentel A S, Alves E S, Alvim R O, Nunes R T, Costa C M A, Lovisi J C M, Lima J R P. Polar $S 810$ como recurso alternativo ao eletrocardiograma no teste de exercício de 4 segundos. Arq Bras Cardiol 2010;94(5):580-4.

http://dx.doi.org/10.1590/S0066-782X2010005000037

8.Rocha RM, Albuquerque DC, Albanesi Filho FM. Variabilidade da frequência cardíaca e ritmo circadiano em pacientes com angina estável. Rev Socerj 2005;18(4):429-42.

9.Barbosa JLR, Belasco DJ. Avaliação da Variabilidade da Frequência Cardíaca em Pacientes com Lesão Medular.Rev Neurocienc 2011;19(2):294-9.

10.Poirier P, Hernandez TL, Weil KM, Shepard TJ, Eckel RH. Impact of Diet-Induced Weight Loss on the Cardiac Autonomic Nervous System in Severe Obesity. Obes Res 2003;11:1040-7.

http://dx.doi.org/10.1038/oby.2003.143

11.Paiva VC, Santana KR, Silva BM, Ramos PS, Lovisi JC, Araújo CG, et al.

Comparaçấo entre métodos de avaliação da modulação vagal cardíaca. Arq Bras

Cardiol 2011;97(6):493-501.

http://dx.doi.org/10.1590/S0066-782X2011005000109

12.Lachish M, Stein D, Kaplan Z, Matar M, Faigin M, Korsunski I, Cohen H. Irreversibility of cardiac autonomic dysfunction in female adolescents diagnosed with anorexia nervosa after short- and long-term weight gain. World J BiolPsychiatry 2009;10(4):503-11.

http://dx.doi.org/10.1080/15622970902980770

13.Ferri R, Curzi-Dascalova L, Arzimanoglou A, Bourgeois M, Beaud C, Nunes $M$, et al. Heart rate variability during sleep in children with partial epilepsy. J Sleep Res 2002;11(2):153.

http://dx.doi.org/10.1046/j.1365-2869.2002.00283.x

14. Ozkaya E, Gursoy E, Demir A, Erenberk U, Sogut A, Dundaroz M R. Autonomic nervous system dysfunction and their relationship with disease severity in children with atopic asthma. Respir Physiol Neurobiol 2012;183: 206-10.

\section{http://dx.doi.org/10.1016/j.resp.2012.07.002}

15.Rajiv KS, Yatan PSB, Rajesh S,Deepak KK, Manju M. Heart rate variability study of childhood anxiety disorders. J Cardiovasc Dis Res 2011;2(2):115-22. http://dx.doi.org/10.4103/0975-3583.83040

16.Godoy MF, Marques GN, Maeda J, Madalozzo BB, Carral LHM,Lorente GD, Sanches KB, Pastre CM, Vanderlei LCM. Gravidade da cardiopatia congênita e nível de redução da variabilidadeda frequência cardíaca. Arq Bras Ciênc Saúde 2012;37(1):19-22.

17.Cambri LT; Oliveira FR; GevaerdMS. Modulação autonômica cardíaca em repouso e controle metabólico em diabéticos tipo 2. HU Revista 2008;34(2):115-21.

18.Angelis K, Santos MSB, Irigoyen MC. Sistema Nervoso Autônomo e Doença Cardiovascular. Revista da Sociedade de Cardiologia do Rio Grande do Sul 2004, p.1-7.

19.Bär K, Letzsch A, Jochum T, Wagner G, Greiner W, Sauer H. Loss of efferent vagal activity in acute schizophrenia. J Psychiatr Res 2005;39(5):519.

http://dx.doi.org/10.1016/j.jpsychires.2004.12.007

20.Chang J, Yoo C, Yi S, Hong K, Lee Y, Oh H, et al. Changes in heart rate dynamics of patients with schizophrenia treated with risperidone. Prog Neuropsychopharmacol Biol Psychiatry 2010;34(6):924.

http://dx.doi.org/10.1016/j.pnpbp.2010.04.017 